Volume 9, No.5, September - October 2020

International Journal of Advanced Trends in Computer Science and Engineering

Available Online at http://www.warse.org/IJATCSE/static/pdf/file/ijatcse307952020.pdf

https://doi.org/10.30534/ijatcse/2020/307952020

\title{
Automated Feature Selection using Boruta Algorithm to Detect Mobile Malware
}

\author{
Che Akmal Che Yahaya, Ahmad Firdaus, Salwana Mohamad, Ferda Ernawan, Mohd Faizal Ab Razak \\ Faculty of Computing, College of Computing and Applied Sciences, University Malaysia Pahang, 26600 Pekan, \\ Pahang Darul Makmur \\ pcn18003@stdmail.ump.edu.my, firdausza@ump.edu.my, salwanamohamad@ump.edu.my, \\ ferda@ump.edu.my, faizalrazak@ump.edu.my
}

\begin{abstract}
The usage of android system is rapidly growing in mobile devices. Android system might also incur severe different malware dangers and security threats such as infections, root exploit, Trojan, and worms. The malware has potential to compromise and steal the private data, classified data, instant messages, private business contacts, and confidential schedule. Malware detection is needed due to the malware continuously evolve rapidly. This research proposed automated feature selection using Boruta algorithm to detect the malware. The proposed method adopts machine learning prediction and optimizes the selecting features in order to reduce the model of machine learning complexity. Boruta algorithm is used to select features automatically for assisting the machine learning. The experimental results show that the proposed method is able to reach $99.73 \%$ accuracy in machine learning classification.
\end{abstract}

Key words: Android; static analysis; machine learning; features.

\section{INTRODUCTION}

Nowadays, people utilize smartphone to communicate and assists them in daily activities, from normal until important tasks. There is various type of operating system (OS) built for smartphone; for instance, Android, IOS, Window, Blackberry and Symbian. Among all these OSes, [1] stated that android is the largest installed platform and growing fast which placed first among others. Consequently, there is a rapid increase in the amount of malware targeting Android smartphones since it is the most popular OS [2].

Malware is an acronym for malicious software - that executes malicious activities such as, secretly accessing private information, causes damage to the OS, or control the system. Therefore, in order to detect malware, security practitioners adopt two types of analyses; dynamic and static. Dynamic analysis is an analysis that execute and monitor the malware behavior [3]. However, dynamic analysis is unable to discover some parts of the code that execute outside of the monitoring range. Apart from that, dynamic analysis is a high resource-consuming analysis, which requires a high specification of hardware [3]. Therefore, static analysis is another alternative for the researcher to detect malware. It is an analysis that studies the malware without executing it. Additionally, this analysis is able to discover the malware that would behave under unusual conditions which is much more accurate [4]. This is due to static analysis examine overall parts of a program including parts that excluded in dynamic analysis, and able to detect unknown malware with machine learning [5].

However, it is important to consider the challenges in machine learning. One of it is the features concern. Higher number of features will decrease the machine learning Android malware detection system performance. This is because by having a large number of features will increase the dimension of search space for the problem. Consequently, this will cause the problem to suffer from Curse of dimensionality [6]. Therefore, there is a need to find a suitable algorithm to select the best among many number of features. Hence, the main contributions in this technical paper are as below:

a) applied Boruta algorithm to select the best features automatically. In author's knowledge, to date, only this paper adopted this algorithm in selecting features in detecting malware for Android platform.

b) this paper discovers the effectiveness of Boruta algorithm in detecting malware.

c) utilized the public dataset [7] which contains hundreds of features that consists of different categories. These features are from 1260 malware and 2539 normal @ benign applications. By using this dataset, Boruta have a wider choice of features to select from and decrease it. From the features selected from the Boruta, it will increase the machine learning detection rate.

d)evaluates the results to measure the Boruta effectiveness to detect malware.

\section{RELATED WORK}

This section introduces the types of malware analysis, and then followed by the machine learning information, types of feature selection, Boruta algorithm and comparison with previous studies. 


\subsection{Malware analysis (dynamic and static)}

There are two types of analysis to detect malware; dynamic and static. Table 1 shows the comparison between these two analyses.

Table 1: Differences between static and dynamic analysis in malware detection

\begin{tabular}{|l|l|l|}
\hline $\begin{array}{c}\text { Type of } \\
\text { analysis }\end{array}$ & \multicolumn{1}{|c|}{ Strength } & \multicolumn{1}{c|}{ Weakness } \\
\hline Dynamic & $\begin{array}{l}\text { 1) May detect anomaly in } \\
\text { malware }\end{array}$ & $\begin{array}{l}\text { 1) Extensive duration } \\
\text { 2) High resource } \\
\text { utilization }\end{array}$ \\
\hline Static & $\begin{array}{l}\text { 1) Instant detection (fast) } \\
\text { 2) May detect malware in } \\
\text { anomaly in malware with } \\
\text { machine learning } \\
\text { prediction. }\end{array}$ & $\begin{array}{l}\text { 1) Need to update the } \\
\text { input data if } \\
\text { needed. }\end{array}$ \\
\hline
\end{tabular}

Dynamic approaches are convenient, yet it does have disadvantages such as high efficiency and profitability. In addition, it is difficult to detect malware types that able to disguise their suspicious activities during analysis. Conversely, static analysis is an approach which evaluates the malicious program without running the applications.

Static (offline) training means that we basically have a big data store and we train our model exactly once before it's used for a long time. Offline training requires less monitoring or monitoring of dataset training work than online training. The downside of the static is that it still requires monitoring at the input data at the inference time, if needed. If our distribution of inputs changes and our model has not adapted, we may end up with a bleak prediction. Hence, it is important to have big data enough to support detection for a long time. However, we able to update the input data anytime as needed as well. Furthermore, with machine learning assist, static analysis able to predict anomaly in malware, similar with dynamic analysis.

\subsection{Machine learning}

Machine learning (ML) is a category of algorithms that allows software applications to become more accurate in predicting outcomes without being explicitly programmed. The basic premise of machine learning is to build algorithms that able to train itself according to the input data given and then use statistical analysis to predict an output. If the new data available, it will update he output. The machine learning classification used in this experiment are Random forest, J48 and GLM. However, machine learning needs relevant features to predict the output efficiently [8][9].

\subsection{Types of feature selection methods}

The selection of features, also known as a selection of variables, selection of attributes, or selection of variable subsets, is the process of selecting a subset of relevant features (variables, predictors) for use in model construction. Feature selection techniques are used for several reasons:

a) Simplification of models to make them easier for researchers/users to interpret.

b) Shorter training time

c) Avoiding the curse of dimensionality

d) Enhanced generalization by reducing over-fitting (formally, reduction of variance)

Accordingly, this paper implements Boruta algorithm to select the best features.

\subsection{Boruta Algorithm}

Boruta is a feature selection algorithm that works as a wrapper built around a random forest classification algorithm. It captures all the important or interesting features of the dataset concerning the output variable. The technique performs a top-down search for important features by comparing the significance of the original attributes with the significant randomly attainable attributes, assessing the use of their permuted duplicates (shadows), and gradually eliminating unimportant features to balance the test. Attributes or features that are significantly better than shadows are recognized as confirmed. On each iteration, shadows are re-created and the algorithm stops when only confirmed features are left. In short, Boruta algorithm use strategy a top-down search for significant features by contrasting original attributes important and important reachable at random, evaluated utilizing their permuted duplicates, and dynamically wiping out irrelevant features to stabilize that test. Therefore, this research uses the Boruta algorithm as a feature selection [10].

\subsection{Comparison of the existing methods}

This section compares the features selection and classification in previous research.

Table 2 lists that, to date, only this experiment used Boruta algorithm in selecting the relevant features in detecting mobile Android, which none researchers apply it previously. In our experiment, Boruta algorithm selects the best features automatically and investigate its effectiveness in selecting the best features.

Table 2: Features selection and classification in previous research

\begin{tabular}{|l|l|l|l|}
\hline $\begin{array}{l}\text { Reference } \\
\text { s }\end{array}$ & Year & $\begin{array}{l}\text { Features Selection } \\
\text { algorithm }\end{array}$ & $\begin{array}{l}\text { Classification } \\
\text { algorithm }\end{array}$ \\
\hline$[11]$ & 2017 & Simulated annealing & Beta Classifier \\
\hline
\end{tabular}




\begin{tabular}{|c|c|c|c|}
\hline & & & $\begin{array}{l}\text { and } \\
\text { Metaclassifier }\end{array}$ \\
\hline [12] & 2018 & Genetic algorithm & $\begin{array}{l}\text { Naïve bayes, } \\
\text { Random forest, } \\
\text { Multilayer } \\
\text { perceptron, J48 } \\
\text { and Functional } \\
\text { tree }\end{array}$ \\
\hline [13] & 2019 & $\begin{array}{l}\text { Permission } \\
\text { ranking-based } \\
\text { features selection } \\
\text { approach }\end{array}$ & Random Forest \\
\hline$[14]$ & 2017 & $\begin{array}{l}\text { Correlation-based } \\
\text { feature selection } \\
\text { (CFS), Chi square } \\
\text { (CHI), Information } \\
\text { gain (IG), ReliefF } \\
\text { (RF) and one wrapper } \\
\text { method with a Linear } \\
\text { SVM classifier (WR) }\end{array}$ & SVM Classifier \\
\hline This paper & $\begin{array}{l}\text { Curren } \\
\text { t year }\end{array}$ & Boruta algorithm & $\mathrm{J} 48$ \\
\hline
\end{tabular}

\section{METHODOLOGY}

This section provides the methodology in conducting our experiment. Figure 1 consists of three phases; 1) literature review; 2) feature selection and machine learning classification; and 3) result.

As Error! Reference source not found. depicts the first phase involves all the review processes that includes identifying the problem statement, objective, scope, existing research, technique to select features and software involves in the experiment. These processes is to achieve the idea and to decide which algorithms need to use to select the best features.

The second phase is where we used $\mathrm{R}$ to import and read the dataset. This paper used a public dataset from a reliable paper entitled "Droidfusion: A Novel Multilevel Classifier
Fusion Approach for Android Malware Detection" [7]. This dataset consists of 1260 malware and 2539 benign@ normal samples. The 215 features in this dataset is based from 3799 Android applications (.apk). The features are form multiple categories, namely; 1) permission; 2) API calls; 3) directory path; and 4) string.

As machine learning need to reduce the complexity of its model, this phase used $\mathrm{R}$ to execute the Boruta algorithm to decrease the number of features automatically. This phase runs Boruta until it reaches a state that there are no features deemed important. Table 3 tabulates 215 features before Boruta algorithm.

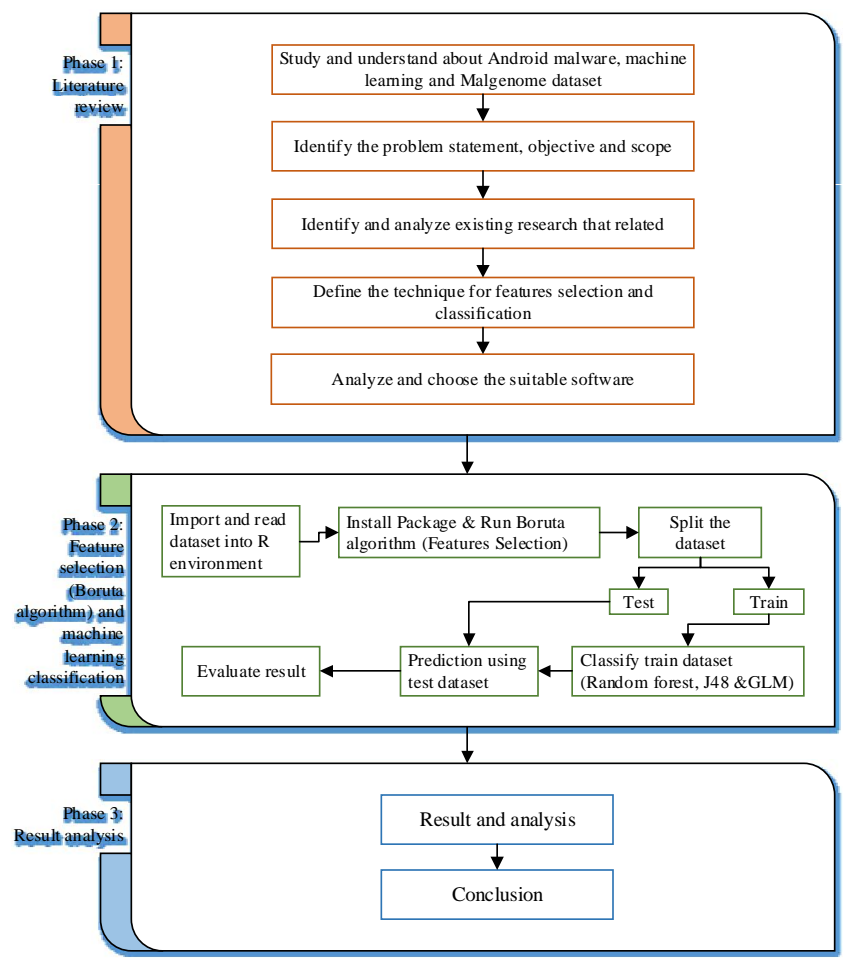

Figure 1: Methodology of Boruta algorithm

Table 3: List of 215 features before Boruta algorithm

\begin{tabular}{|c|c|c|c|c|c|}
\hline transact & Ljava.lang.Class.forName & READ_SYNC_STATS & DexClassLoader & intent.action.RUN & STATUS_BAR \\
\hline bindService & $\begin{array}{l}\text { TelephonyManager.getSimSer } \\
\text { ialNumber }\end{array}$ & $\begin{array}{l}\text { WRITE_HISTORY_BOOK } \\
\text { MARKS }\end{array}$ & WRITE_CALENDAR & SecretKey & Ljavax.crypto.Cipher \\
\hline onServiceConnected & CAMERA & DISABLE_KEYGUARD & $\begin{array}{l}\text { PROCESS_OUTGOING_C } \\
\text { ALLS }\end{array}$ & CLEAR_APP_CACHE & $\begin{array}{l}\text { MODIFY_PHONE_STA } \\
\text { TE }\end{array}$ \\
\hline ServiceConnection & CALL_PHONE & READ_LOGS & BIND_DEVICE_ADMIN & $\begin{array}{l}\text { ACCESS_FINE_LOCATI } \\
\text { ON }\end{array}$ & $\begin{array}{l}\text { android.intent.action.PA } \\
\text { CKAGE_RESTARTED }\end{array}$ \\
\hline android.os.Binder & android.intent.action.SEND & RECORD_AUDIO & $\begin{array}{l}\text { CHANGE_WIFI_MULTIC } \\
\text { AST_STATE }\end{array}$ & $\begin{array}{l}\text { SET_WALLPAPER_HIN } \\
\text { TS }\end{array}$ & READ_INPUT_STATE \\
\hline READ_SMS & onBind & getCallingPid & MASTER_CLEAR & Context.bindService & $\begin{array}{l}\text { READ_EXTERNAL_S } \\
\text { TORAGE }\end{array}$ \\
\hline attachInterface & android.content.pm.Signature & $\begin{array}{l}\text { MODIFY_AUDIO_SETTI } \\
\text { NGS }\end{array}$ & $\begin{array}{l}\text { android.intent.action.PACK } \\
\text { AGE_DATA_CLEARED }\end{array}$ & MessengerService & $\begin{array}{l}\text { Ljava.lang.Object.getClas } \\
\mathrm{s}\end{array}$ \\
\hline WRITE_SMS & READ_SYNC_SETTINGS & $\begin{array}{l}\text { android.intent.action.PACK } \\
\text { AGE_REPLACED }\end{array}$ & FLASHLIGHT & $\begin{array}{l}\text { ACCESS_NETWORK_S } \\
\text { TATE }\end{array}$ & SET_ORIENTATION \\
\hline $\begin{array}{l}\text { TelephonyManager.getSubscri } \\
\text { berId }\end{array}$ & $\begin{array}{l}\text { AUTHENTICATE_ACCOU } \\
\text { NTS }\end{array}$ & $\begin{array}{l}\text { android.intent.action.TIMEZ } \\
\text { ONE_CHANGED }\end{array}$ & $\begin{array}{l}\text { android.intent.action.BATT } \\
\text { ERY_LOW }\end{array}$ & $\begin{array}{l}\text { android.content.pm.Packag } \\
\text { eInfo }\end{array}$ & DEVICE_POWER \\
\hline $\begin{array}{l}\text { Ljava.lang.Class.getCanonical } \\
\text { Name }\end{array}$ & INTERNET & BROADCAST_STICKY & SET_ALARM & $\begin{array}{l}\text { BIND_ACCESSIBILITY } \\
\text { _SERVICE }\end{array}$ & $\begin{array}{l}\text { EXPAND_STATUS_B } \\
\text { AR }\end{array}$ \\
\hline Ljava.lang.Class.getMethods & PackageInstaller & Runtime.exec & RECEIVE_MMS & $\begin{array}{l}\text { INTERNAL_SYSTEM_ } \\
\text { WINDOW }\end{array}$ & GET_TASKS \\
\hline $\begin{array}{l}\text { android.intent.action.BOOT_C } \\
\text { OMPLETED }\end{array}$ & $\begin{array}{l}\text { ACCESS_LOCATION_EXT } \\
\text { RA_COMMANDS }\end{array}$ & $\begin{array}{l}\text { android.intent.action.PACK } \\
\text { AGE_ADDED }\end{array}$ & divideMessage & SET_TIME_ZONE & GLOBAL_SEARCH \\
\hline
\end{tabular}


Che Akmal Che Yahaya et al., International Journal of Advanced Trends in Computer Science and Engineering, 9(5),September-October 2020,9029 - 9036

\begin{tabular}{|c|c|c|c|c|c|}
\hline Ljava.lang.Class.getField & HttpUriRequest & $\begin{array}{l}\text { MOUNT_UNMOUNT_FIL } \\
\text { ESYSTEMS }\end{array}$ & WRITE_CALL_LOG & Process.start & GET_PACKAGE_SIZE \\
\hline READ_PHONE_STATE & remount & $\begin{array}{l}\text { android.intent.action.ACTIO } \\
\text { N_POWER_DISCONNEC } \\
\text { TED }\end{array}$ & WRITE_PROFILE & $\begin{array}{l}\text { MOUNT_FORMAT_FIL } \\
\text { ESYSTEMS }\end{array}$ & $\begin{array}{l}\text { SET_PREFERRED_AP } \\
\text { PLICATIONS }\end{array}$ \\
\hline $\begin{array}{l}\begin{array}{l}\text { Landroid.content.Context.unre } \\
\text { gisterReceiver }\end{array} \\
\end{array}$ & $\begin{array}{l}\text { android.telephony.SmsManage } \\
\text { r }\end{array}$ & $\begin{array}{l}\text { Ljava.lang.Class.getDeclared } \\
\text { Classes }\end{array}$ & $\begin{array}{l}\text { WRITE_USER_DICTION } \\
\text { ARY }\end{array}$ & $\begin{array}{l}\text { CLEAR_APP_USER_DA } \\
\text { TA }\end{array}$ & $\begin{array}{l}\text { android.intent.action.PA } \\
\text { CKAGE_CHANGED }\end{array}$ \\
\hline GET_ACCOUNTS & $\begin{array}{l}\text { RECEIVE_BOOT_COMPLE } \\
\text { TED }\end{array}$ & $\begin{array}{l}\text { android.intent.action.PACK } \\
\text { AGE_REMOVED }\end{array}$ & BIND_INPUT_METHOD & $\begin{array}{l}\text { UPDATE_DEVICE_STA } \\
\text { TS }\end{array}$ & \\
\hline SEND_SMS & $\begin{array}{l}\text { android.intent.action.ACTION } \\
\text { POWER_CONNECTED }\end{array}$ & BLUETOOTH_ADMIN & READ_SOCIAL_STREAM & IRemoteService & \\
\hline $\begin{array}{l}\text { Landroid.content.Context.regis } \\
\text { terReceiver }\end{array}$ & findClass & android.os.IBinder & REORDER_TASKS & $\begin{array}{l}\text { android.intent.action.SET_ } \\
\text { WALLPAPER }\end{array}$ & \\
\hline getBinder & WRITE_CONTACTS & IBinder & defineClass & $\begin{array}{l}\text { BROADCAST_WAP_PU } \\
\text { SH }\end{array}$ & \\
\hline Ljava.lang.Class.cast & .system.app & $\begin{array}{l}\text { WRITE_SECURE_SETTI } \\
\text { NGS }\end{array}$ & PERSISTENT_ACTIVITY & $\begin{array}{l}\text { android.intent.action.CALL } \\
\text { BUTTON }\end{array}$ & \\
\hline chmod & Ljava.lang.Class.getResource & WRITE_SETTINGS & ProcessBuilder & INJECT_EVENTS & \\
\hline createSubprocess & WRITE_SYNC_SETTINGS & $\begin{array}{l}\text { Ljavax.crypto.spec.SecretKe } \\
\text { ySpec }\end{array}$ & $\begin{array}{l}\text { android.intent.action.SCREE } \\
\text { N_ON }\end{array}$ & $\begin{array}{l}\text { ACCESS_SURFACE_FLI } \\
\text { NGER }\end{array}$ & \\
\hline Ljava.net.URLDecoder & $\begin{array}{l}\text { android.intent.action.TIME_S } \\
\text { ET }\end{array}$ & $\begin{array}{l}\text { android.intent.action.BATT } \\
\text { ERY_OKAY }\end{array}$ & $\begin{array}{l}\text { READ_USER_DICTIONA } \\
\text { RY }\end{array}$ & SET_PROCESS_LIMIT & \\
\hline WRITE_APN_SETTINGS & $\begin{array}{l}\text { android.intent.action.SEND_ } \\
\text { MULTIPLE }\end{array}$ & READ_CONTACTS & $\begin{array}{l}\text { WRITE_SOCIAL_STREA } \\
\text { M }\end{array}$ & ADD_VOICEMAIL & \\
\hline $\begin{array}{l}\text { TelephonyManager.getDeviceI } \\
\text { d }\end{array}$ & ACCESS_WIFI_STATE & Binder & SET_TIME & $\begin{array}{l}\text { INSTALL_LOCATION_P } \\
\text { ROVIDER }\end{array}$ & \\
\hline RECEIVE_SMS & URLClassLoader & $\begin{array}{l}\text { SUBSCRIBED_FEEDS_R } \\
\text { EAD }\end{array}$ & mount & $\begin{array}{l}\text { SET_ACTIVITY_WATC } \\
\text { HER }\end{array}$ & \\
\hline $\begin{array}{l}\text { Ljava.lang.Class.getDeclaredFi } \\
\text { eld }\end{array}$ & BLUETOOTH & READ_CALL_LOG & System.loadLibrary & $\begin{array}{l}\text { TelephonyManager.getCall } \\
\text { State }\end{array}$ & \\
\hline HttpGet.init & WAKE_LOCK & $\begin{array}{l}\text { SUBSCRIBED_FEEDS_W } \\
\text { RITE }\end{array}$ & $\begin{array}{l}\text { CHANGE_COMPONENT_ } \\
\text { ENABLED_STATE }\end{array}$ & VIBRATE & \\
\hline Ljava.lang.Class.getPackage & $\begin{array}{l}\text { SYSTEM_ALERT_WINDO } \\
\text { W }\end{array}$ & BATTERY_STATS & $\begin{array}{l}\text { ACCESS_MOCK_LOCATI } \\
\text { ON }\end{array}$ & Runtime.getRuntime & \\
\hline abortBroadcast & $\begin{array}{l}\text { TelephonyManager.getSimCo } \\
\text { untryIso }\end{array}$ & RECEIVE_WAP_PUSH & DUMP & $\begin{array}{l}\text { CHANGE_CONFIGURA } \\
\text { TION }\end{array}$ & \\
\hline ClassLoader & chown & PathClassLoader & CALL_PRIVILEGED & BROADCAST_SMS & \\
\hline $\begin{array}{l}\text { TelephonyManager.getLine1N } \\
\text { umber }\end{array}$ & NFC & $\begin{array}{l}\text { KILL_BACKGROUND_PR } \\
\text { OCESSES }\end{array}$ & DELETE_PACKAGES & BIND_WALLPAPER & \\
\hline getCallingUid & $\begin{array}{l}\text { READ_HISTORY_BOOKM } \\
\text { ARKS }\end{array}$ & $\begin{array}{l}\text { ACCESS_COARSE_LOCA } \\
\text { TION }\end{array}$ & READ_FRAME_BUFFER & $\begin{array}{l}\text { BROADCAST_PACKAG } \\
\text { E REMOVED }\end{array}$ & \\
\hline USE_CREDENTIALS & HttpPost.init & $\begin{array}{l}\text { android.intent.action.ACTIO } \\
\text { N_SHUTDOWN }\end{array}$ & WRITE_GSERVICES & $\begin{array}{l}\text { TelephonyManager.isNetw } \\
\text { orkRoaming }\end{array}$ & \\
\hline MANAGE_ACCOUNTS & $\begin{array}{l}\text { TelephonyManager.getNetwor } \\
\text { kOperator }\end{array}$ & Runtime.load & ACCOUNT_MANAGER & $\begin{array}{l}\text { TelephonyManager.getSim } \\
\text { Operator }\end{array}$ & \\
\hline $\begin{array}{l}\text { android.telephony.gsm.SmsMa } \\
\text { nager }\end{array}$ & Ljava.lang.Class.getClasses & $\begin{array}{l}\text { android.intent.action.SEND } \\
\text { TO }\end{array}$ & KeySpec & $\begin{array}{l}\text { WRITE_EXTERNAL_ST } \\
\text { ORAGE }\end{array}$ & \\
\hline .system.bin & BIND_REMOTEVIEWS & $\begin{array}{l}\text { SET_WALLPAPER } \\
\end{array}$ & sendDataMessage & $\begin{array}{l}\text { android.intent.action.CAM } \\
\text { ERA_BUTTON }\end{array}$ & \\
\hline Ljava.lang.Class.getMethod & READ_PROFILE & $\begin{array}{l}\text { android.intent.action.NEW } \\
\text { OUTGOING_CALL }\end{array}$ & android.intent.action.CALL & $\begin{array}{l}\text { android.intent.action.REB } \\
\text { OOT }\end{array}$ & \\
\hline RESTART_PACKAGES & READ_CALENDAR & $\begin{array}{l}\text { CHANGE_NETWORK_ST } \\
\text { ATE }\end{array}$ & BIND_APPWIDGET & sendMultipartTextMessage & \\
\hline INSTALL_PACKAGES & CHANGE_WIFI_STATE & REBOOT & $\begin{array}{l}\text { android.intent.action.SCREE } \\
\text { N_OFF }\end{array}$ & BIND_VPN_SERVICE & \\
\hline
\end{tabular}




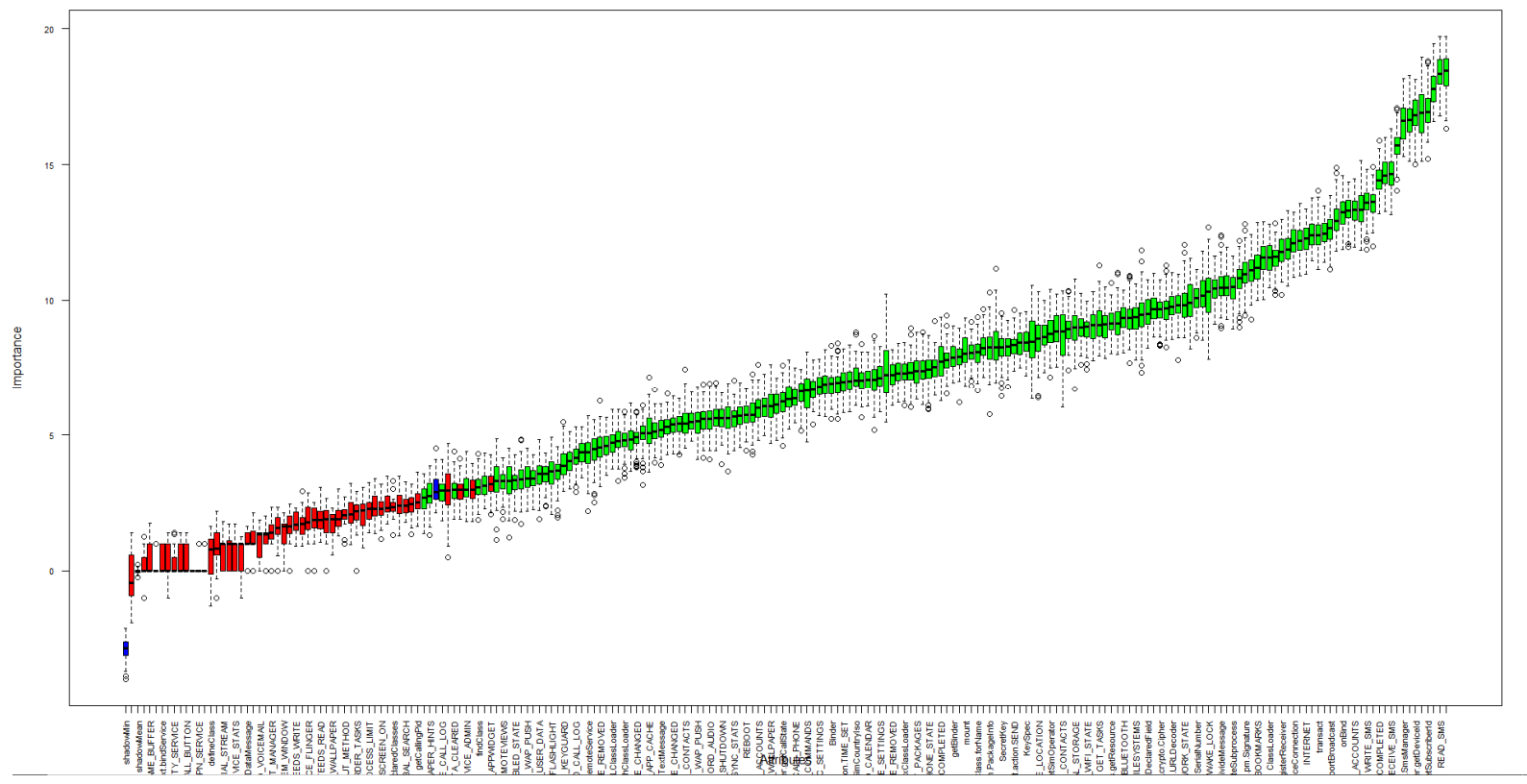

Figure 2: 1st round of Boruta

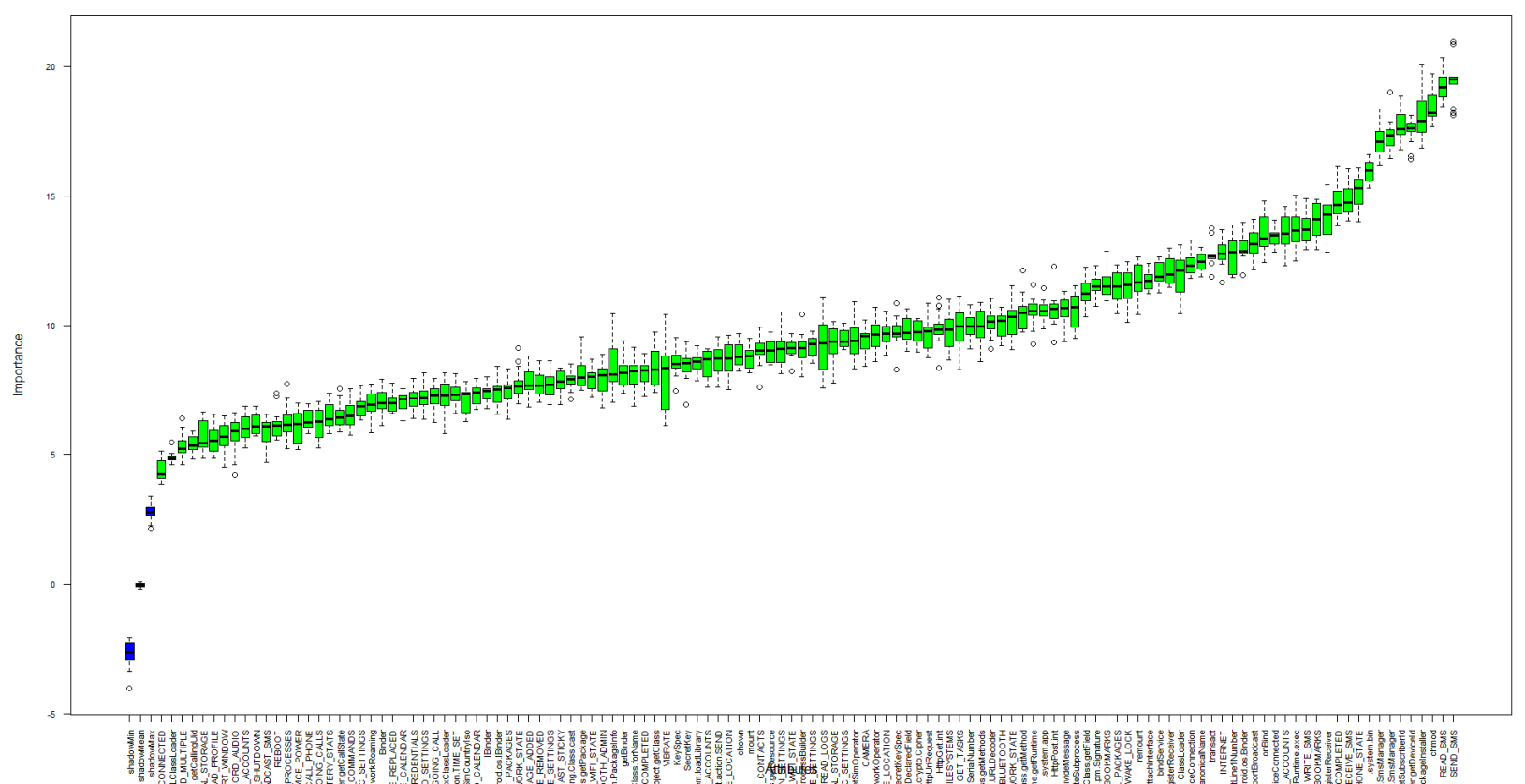

Figure 3: 8 th round of Boruta

Table 4: List of 124 features after Boruta algorithm

\begin{tabular}{|c|c|c|c|c|}
\hline transact & RECEIVE_SMS & PackageInstaller & $\begin{array}{l}\text { WRITE_HISTORY_BOOKM } \\
\text { ARKS }\end{array}$ & DexClassLoader \\
\hline bindService & $\begin{array}{l}\text { Ljava.lang.Class.getDeclaredF } \\
\text { ield }\end{array}$ & $\begin{array}{l}\text { ACCESS_LOCATION_EXT } \\
\text { RA_COMMANDS }\end{array}$ & READ_LOGS & WRITE_CALENDAR \\
\hline onServiceConnected & HttpGet.init & HttpUriRequest & RECORD_AUDIO & $\begin{array}{l}\text { PROCESS_OUTGOING_CA } \\
\text { LLS }\end{array}$ \\
\hline ServiceConnection & Ljava.lang.Class.getPackage & remount & $\begin{array}{l}\text { MODIFY_AUDIO_SETTING } \\
\text { S }\end{array}$ & divideMessage \\
\hline android.os.Binder & abortBroadcast & $\begin{array}{l}\text { android.telephony.SmsManage } \\
\mathrm{r}\end{array}$ & $\begin{array}{l}\text { android.intent.action.PACKAG } \\
\text { E_REPLACED }\end{array}$ & ProcessBuilder \\
\hline READ_SMS & ClassLoader & $\begin{array}{l}\text { RECEIVE_BOOT_COMPLE } \\
\text { TED }\end{array}$ & BROADCAST_STICKY & mount \\
\hline attachInterface & TelephonyManager.getLine1N & android.intent.action.ACTION & Runtime.exec & System.loadLibrary \\
\hline
\end{tabular}




\begin{tabular}{|c|c|c|c|c|}
\hline & umber & _POWER_CONNECTED & & \\
\hline WRITE_SMS & getCallingUid & .system.app & $\begin{array}{l}\text { android.intent.action.PACKAG } \\
\text { E_ADDED }\end{array}$ & KeySpec \\
\hline $\begin{array}{l}\text { TelephonyManager.getS } \\
\text { ubscriberId }\end{array}$ & USE_CREDENTIALS & Ljava.lang.Class.getResource & $\begin{array}{l}\text { MOUNT_UNMOUNT_FILES } \\
\text { YSTEMS }\end{array}$ & SecretKey \\
\hline $\begin{array}{l}\text { Ljava.lang.Class.getCano } \\
\text { nicalName }\end{array}$ & MANAGE_ACCOUNTS & WRITE_SYNC_SETTINGS & $\begin{array}{l}\text { android.intent.action.PACKAG } \\
\text { E_REMOVED }\end{array}$ & ACCESS_FINE_LOCATION \\
\hline $\begin{array}{l}\text { Ljava.lang.Class.getMeth } \\
\text { ods }\end{array}$ & $\begin{array}{l}\text { android.telephony.gsm.SmsMa } \\
\text { nager }\end{array}$ & $\begin{array}{l}\text { android.intent.action.TIME_S } \\
\text { ET }\end{array}$ & BLUETOOTH_ADMIN & $\begin{array}{l}\text { ACCESS_NETWORK_STAT } \\
\text { E }\end{array}$ \\
\hline $\begin{array}{l}\text { android.intent.action.BO } \\
\text { OT_COMPLETED }\end{array}$ & .system.bin & $\begin{array}{l}\text { android.intent.action.SEND_ } \\
\text { MULTIPLE }\end{array}$ & android.os.IBinder & $\begin{array}{l}\text { android.content.pm.PackageInf } \\
\text { o }\end{array}$ \\
\hline Ljava.lang.Class.getField & Ljava.lang.Class.getMethod & ACCESS_WIFI_STATE & IBinder & $\begin{array}{l}\text { TelephonyManager.getCallSta } \\
\text { te }\end{array}$ \\
\hline $\begin{array}{l}\text { READ_PHONE_STAT } \\
\text { E }\end{array}$ & RESTART_PACKAGES & URLClassLoader & WRITE_SECURE_SETTINGS & VIBRATE \\
\hline $\begin{array}{l}\text { Landroid.content.Context } \\
\text {.unregisterReceiver }\end{array}$ & INSTALL_PACKAGES & BLUETOOTH & WRITE_SETTINGS & Runtime.getRuntime \\
\hline GET_ACCOUNTS & Ljava.lang.Class.forName & WAKE_LOCK & $\begin{array}{l}\text { Ljavax.crypto.spec.SecretKeySp } \\
\text { ec }\end{array}$ & BROADCAST_SMS \\
\hline SEND_SMS & $\begin{array}{l}\text { TelephonyManager.getSimSeri } \\
\text { alNumber }\end{array}$ & $\begin{array}{l}\text { SYSTEM_ALERT_WINDO } \\
\text { W }\end{array}$ & READ_CONTACTS & $\begin{array}{l}\text { TelephonyManager.isNetwork } \\
\text { Roaming }\end{array}$ \\
\hline $\begin{array}{l}\text { Landroid.content.Context } \\
\text {.registerReceiver }\end{array}$ & CAMERA & $\begin{array}{l}\text { TelephonyManager.getSimCo } \\
\text { untryIso }\end{array}$ & Binder & $\begin{array}{l}\text { TelephonyManager.getSimOp } \\
\text { erator }\end{array}$ \\
\hline getBinder & CALL_PHONE & chown & BATTERY_STATS & $\begin{array}{l}\text { WRITE_EXTERNAL_STOR } \\
\text { AGE }\end{array}$ \\
\hline Ljava.lang.Class.cast & android.intent.action.SEND & $\begin{array}{l}\text { READ_HISTORY_BOOKM } \\
\text { ARKS }\end{array}$ & \begin{tabular}{|l|} 
KILL_BACKGROUND_PRO \\
CESSES
\end{tabular} & Ljavax.crypto.Cipher \\
\hline chmod & onBind & HttpPost.init & $\begin{array}{l}\text { ACCESS_COARSE_LOCATI } \\
\text { ON }\end{array}$ & $\begin{array}{l}\text { READ_EXTERNAL_STORA } \\
\text { GE }\end{array}$ \\
\hline createSubprocess & android.content.pm.Signature & $\begin{array}{l}\text { TelephonyManager.getNetwor } \\
\text { kOperator }\end{array}$ & $\begin{array}{l}\text { android.intent.action.ACTION } \\
\text { SHUTDOWN }\end{array}$ & Ljava.lang.Object.getClass \\
\hline Ljava.net.URLDecoder & READ_SYNC_SETTINGS & READ_PROFILE & $\begin{array}{l}\text { android.intent.action.NEW_OU } \\
\text { TGOING_CALL }\end{array}$ & DEVICE_POWER \\
\hline $\begin{array}{l}\text { WRITE_APN_SETTIN } \\
\text { GS }\end{array}$ & $\begin{array}{l}\text { AUTHENTICATE_ACCOU } \\
\text { NTS }\end{array}$ & READ_CALENDAR & $\begin{array}{l}\text { CHANGE_NETWORK_STAT } \\
\text { E }\end{array}$ & GET_TASKS \\
\hline $\begin{array}{l}\text { TelephonyManager.getD } \\
\text { eviceId }\end{array}$ & INTERNET & CHANGE_WIFI_STATE & REBOOT & \\
\hline
\end{tabular}

This phase runs from $1^{\text {st }}$ to $8^{\text {th }}$ round of Boruta features. Figure 2 shows the result of the Boruta in $1^{\text {st }}$ round, while the following figure (Figure 3) depicts the $8^{\text {th }}$ round. The number of times to reach this state is $8^{\text {th }}$ times. Once the process is finished, the Boruta successfully reduced the features from 215 to 124 . Table 4 lists those 124 features. With these 124 features, this phase trains three machine learning classifiers (Random forest, J48 and GLM) to construct a model. Then, in order to test the model either it able to detect unknown malware, we test this model with the data that excluded from the training part. In phase three, we executed the classification process. The machine learning classifier used are Random Forest, J48, and GLM.

\section{EXPERIMENTAL RESULTS}

This section discussed the results of machine learning classification, derived from the Boruta algorithm. The following figures depict the results of the classification.

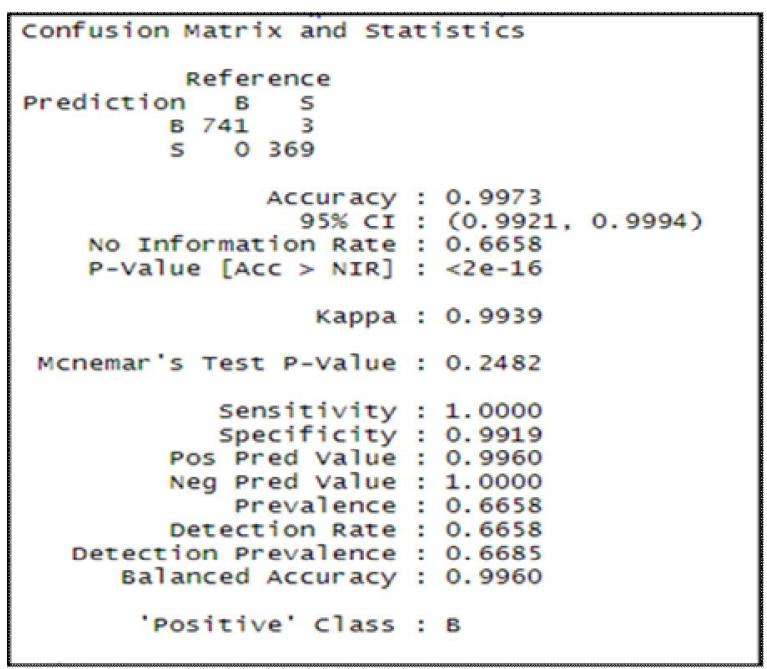

Figure 4: Random Forest algorithm result

Random Forest algorithm is one of the methods used in this research for the classification process. Figure 4 shows the result of the Random Forest classifier. algorithm predicts the class of the data. There are two classes which are ' $\mathrm{B}$ ' for Benign and ' $S$ ' it shows the confusion matrix and statistics for the Random Forest algorithm using the test dataset. There are 1140 data inside the test dataset. Random Forest's accuracy is 0.9973 . 


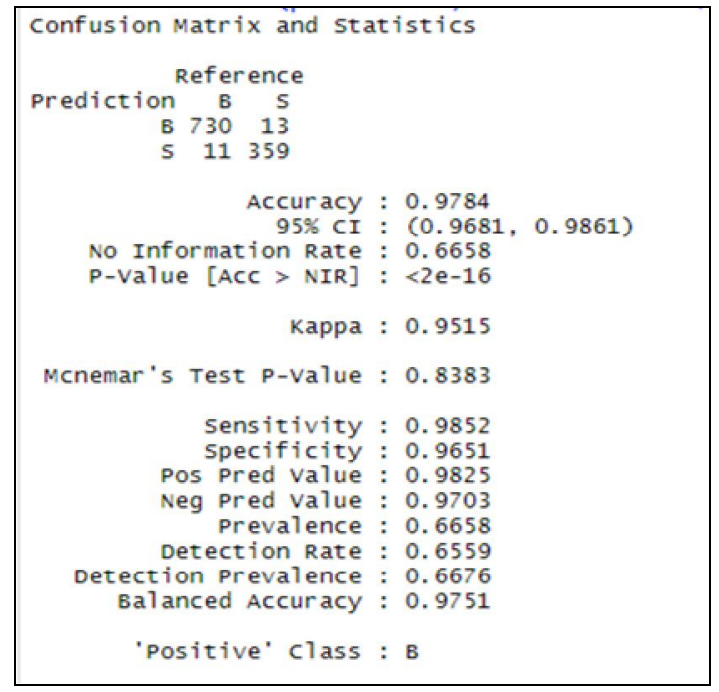

Figure 5: J48 algorithm result

Figure 5 shows the confusion matrix and statistics for the J48 algorithm using the Test dataset. There are 1140 data inside the test dataset. J48 accuracy is 0.9784 .

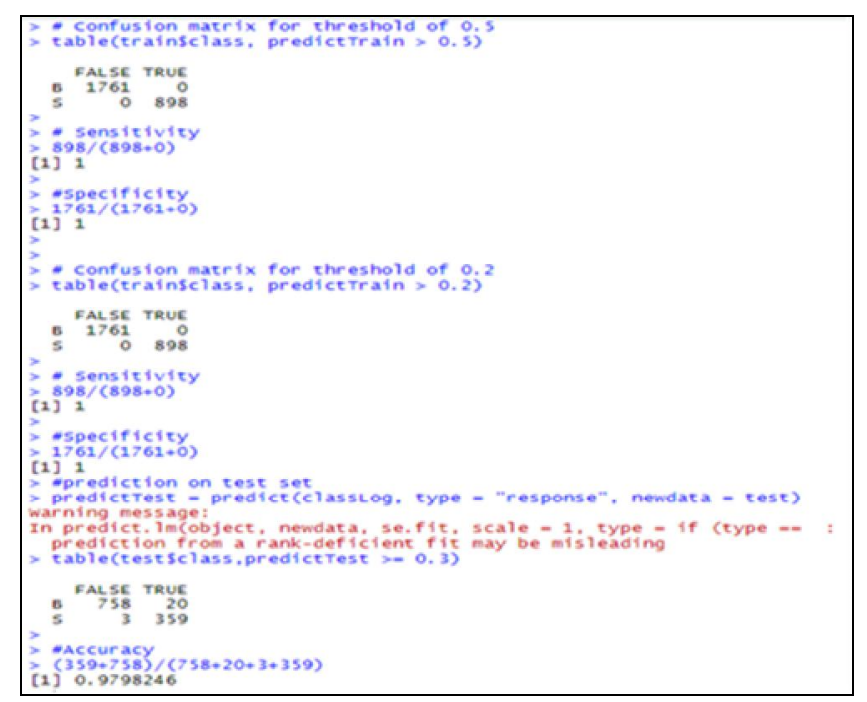

Figure 6: GLM algorithm result

Error! Reference source not found. shows the GLM confusion matrix algorithm using the test dataset. There are 1140 data in the test dataset. The precision is 0.9798 . The GLM sensitivity and specificity are calculated manually based on the confusion matrix that is generated automatically by the system. Table below list overall accuracies with different machine learning classifiers.

Table 5: Result accuracy each algorithm

\begin{tabular}{|l|l|}
\hline Classifiers & Accuracy \\
\hline Random forest & 0.9973 \\
\hline J48 & 0.9784 \\
\hline GLM & 0.9798 \\
\hline
\end{tabular}

Table 5 both classifier which is Random Forest and J48 decision tree, it shows that Random Forest has the higher accuracy which is 0.99 while J48 Decision Tree is 0.97 . In conclusion, Random Forest is the best compare to J48 and GLM.

\section{CONCLUSION}

Malware continuously evolve rapidly and it also compromise and steal the private data especially in Android system. Malware detection with high accuracy is needed to detect its malicious activities. This paper presented automated feature selection using boruta algorithm and machine learning method to detect and predict mobile malware. The proposed the optimal features selection is able to reduce the model of machine learning complexity. The experimental results show that the proposed Boruta algorithm provides automatically feature selection for increasing the machine learning detection. The result shows that random forest successfully achieved $99.73 \%$ accuracy in machine learning detection. The proposed scheme able to select the best and relevant features efficiently.

\section{ACKNOWLEDGEMENT}

This work is supported by Ministry of Higher Education (MOHE) for Fundamental Research Grant Scheme (FRGS-RACER) with grant number RDU192607, RACER/1/2019/ICT02/UMP//5, and Universiti Malaysia Pahang, under the Grant IBM Centre of Excellence (COE)(IBM2000), RDU180337.

\section{REFERENCES}

1. F. M. Gotz, S. Stieger, and U. D. Reips. Users of the main smartphone operating systems (iOS, Android) differ only little in personality, PLOS ONE, vol. 12, no. 5, pp. 1-18, 2017.

2. M. F. A. Razak, N. B. Anuar, R. Salleh, and A. Firdaus. The rise of "malware": Bibliometric analysis of malware study, Journal of Network and Computer Applications, vol. 75, pp. 58-76, 2016.

3. W. Enck. Defending Users against Smartphone Apps: Techniques and Future Directions, Proceedings of the 7 th international conference on Information Systems Security, pp. 49-70, 2011.

4. C. A. Castillo. Android Malware Past, Present, and Future, McAfee White Paper, Mobile Security Working Group, 2011. [Online]. Available: http://www.mcafee.com/us/ resources/white-papers/wp-android-malware-past-prese nt-future.pdf.

5. K. Tam, A. Feizollah, N. B. Anuar, R. Salleh, and L. Cavallaro. The Evolution of Android Malware and Android Analysis Techniques, ACM Computing Surveys (CSUR), vol. 49, no. 4, pp. 1-41, 2017.

6. A. Kumar, K. S. Kuppusamy, and G. Aghila. FAMOUS: Forensic Analysis of Mobile Devices Using Scoring of 
application permissions, Future Generation Computer Systems, vol. 83, pp. 158-172, 2018.

7. S. Y. Yerima and S. Sezer. DroidFusion: A Novel Multilevel Classifier Fusion Approach for Android Malware Detection, IEEE Transactions on Cybernetics, vol. 49, no. 2, pp. 453-466, 2019.

8. A. Feizollah, N. B. Anuar, R. Salleh, and A. W. A. Wahab. A review on feature selection in mobile malware detection, Digital Investigation, vol. 13, pp. 22-37, 2015.

9. A. Firdaus, N. B. Anuar, M. F. A. Razak, I. A. T. Hashem, S. Bachok, and A. K. Sangaiah. Root Exploit Detection and Features Optimization: Mobile Device and Blockchain Based Medical Data Management, Journal of Medical Systems, vol. 42, no. 112, pp. 1-23, 2018.

10. M. B. Kursa, A. Jankowski, and W. R. Rudnicki. Boruta-A system for feature selection, Fundamenta Informaticae, vol. 101, no. 4, pp. 271-285, 2010.

11. M. Hassen and P. K. Chan. Scalable function call graph-based malware classification, Proceedings of the 7th ACM Conference on Data and Application Security and Privacy, pp. 239-248, 2017.

12. A. Firdaus, N. B. Anuar, A. Karim, and M. F. A. Razak. Discovering optimal features using static analysis and a genetic search based method for Android malware detection, Frontiers of Information Technology and Electronic Engineering, vol. 19, no. 6, pp. 712-736, Jun. 2018.

13. R. Kumar, X. Zhang, R. U. Khan, and A. Sharif. Research on data mining of permission-induced risk for android IoT devices, Applied Sciences, vol. 9, no. 2, pp. 1-22, 2019.

14. M. Z. Mas'ud, S. Sahib, M. F. Abdollah, S. R. Selamat, and C. Y. Huoy. A comparative study on feature selection method for N-gram mobile malware detection, International Journal of Network Security, vol. 19, no. 5, pp. 727-733, 2017. 